Article original

\title{
La fonction thyroïdienne au cours du premier trimestre de la grossesse
}

\author{
Thyroid function in the first trimester of pregnancy
}

\author{
Hanachi Sabah ${ }^{1,2}$, Sifi Karima ${ }^{1,2}$.Bouchedjera Yamina', Boudaoud Khalida3, Zekri Salima1,2, Benem- \\ barek Karima ${ }^{1,2}$, Abadi.Noureddine ${ }^{1}$ \\ ${ }^{1}$ Laboratoire de biochimie CHU Constantine \\ ¿Laboratoire de biologie et génétique moléculaire, Faculté de médecine, Université Salah Boubnider Constantine3 \\ ${ }^{3}$ Sevice d'endocrinologie CHU Constantine
}

Auteur correspondant: hanachisabah@yahoo.fr

Article reçu le 11/11/2019 Accepté le 18/12/2019

\section{MOTS CLÉS}

Thyroïde, premier trimestre de la grossesse, fT3, fT4, TSH.

\section{Résumé}

Introduction - Les dysthyroïdies représentent les secondes pathologies les plus fréquentes après le diabète chez la femme enceinte.

La thyroïde subit de nombreux changements physiologiques pendant la grossesse et son activité fonctionnelle augmente de $50 \%$. Les hormones thyroïdiennes jouent un rôle important dans les premiers stades du développement du cerveau. Au cours du premier trimestre, le fœtus est dépendant de la production d'hormones thyrö̈diennes maternelles. Il est donc important de détecter, typer et éventuellement traiter toute anomalie thyroïdienne au début de la grossesse; l'idéal étant bien évidemment une prise en charge de ces pathologies avant la conception.

Patients et méthodes - Dans ce travail, nous avons étudié les variations des concentrations plasmatiques des paramètres du bilan thyroïdien à savoir l'hormone thyréostimulante (TSH), la thyroxine libre (fT4) et tri-iodothyronine libre (fT3) chez cinquante gestantes au 1ertrimestre de la grossesse.

Résultats - Parmi les cinquante femmes enceintes au 1er trimestre concernées par notre étude, 11 étaient au 1er mois, 19 au Lème et 20 au 3ème mois de grossesse. L'âge moyen de nos gestantes était de 30 $\pm 6,76$ ans avec des extrêmes allant de 17 à 45 ans. Le dosage de la TSH a révélé une valeur moyenne chez nos gestantes de 2,37 4 4,01 $\mathrm{mUI} / \mathrm{L}$ avec des extrêmes allant de 0,031 à 26,37mUI/L. La prévalence de l'hypothyroïdie dans notre série était de $8 \%$. Les deux autres paramètres du bilan thyroïdien (fT3, fT4) ont présenté une élévation de leurs concentrations au premier mois puis une stabilisation au Zème et 3ème mois de grossesse.

Conclusion - Les dysthyroïdies sont fréquentes dans notre population d'étude, elles doivent être systématiquement recherchées chez toute femme enceinte et correctement prises en charge afin de prévenir les complications à la fois maternelles et fœtales. 


\section{KEY WORDS}

Thyroid, first trimester of pregnancy, fT3, fT4, TSH.

\section{Introduction}

Les dysthyroïdies représentent les secondes pathologies les plus fréquentes après le diabète chez la femme enceinte [1], et l'obstétricien est fréquemment amené à suivre ces grossesses conjointement avec l'endocrinologue. La thyroïde subit de nombreux changements physiologiques pendant la grossesse. L'activité fonctionnelle de la glande est augmentée avec une hausse de $50 \%$ de la production des hormones thyroïdiennes [2]. Ces hormones thyroïdiennes ont un rôle important dès les premières étapes du développement du cerveau chez l'homme. Pendant le premier trimestre, le fœtus est dépendant de cette production hormonale maternelle [3].

Durant la grossesse, les variations physiologiques de la fonction thyroïdienne sont facilement décompensées par une carence iodée même très modeste. De plus, l'hyper comme l'hyopthyroïdie ont des conséquences non seulement sur le déroulement de la grossesse, mais aussi sur le fœtus [4]. En effet, depuis les deux dernières décennies, les recherches ont identifié de multiples conséquences délétères affectant la mère et le fœtus associées aux anomalies thyroïdiennes et à l'auto immunité thyroïdienne maternelle. C'est en 1990, que pour la première fois Stagnaro Green et al, ont montré une association entre la présence des anticorps antithyroïdiens et les fausses couches [5]. En 1999 Haddow et al ont été les premiers à montrer une diminution des performances intellectuelles chez les enfants nés de mères ayant eu une hypothyroïdie pendant la grossesse $[6,7]$. L'hypothyroïdie a également des conséquences délétères obstétricales, telles que une hypertension gravidique, une pré éclampsie, une anémie, un avortement et des hémorragies du postpartum et d'autres conséquences fœtales comme un retard de croissance intra utérin, une morbidité et mortalité fœtale et périnatale accrue; tout comme l'hyperthyroïdie [4].

La fréquence de la pathologie thyroïdienne chez la femme jeune confronte régulièrement l'obstétricien au suivi de la grossesse de patientes ayant une dysthyroïdie de sévérité variable. Les potentielles répercussions fœto-maternelles du dysfonctionnement thyroïdien pendant la grossesse justifient une étroite collaboration entre obstétricien et endocrinologue, afin d'assurer aux patientes une prise en charge précoce et adaptée.

Il est donc important de détecter, typer et éventuellement 
traiter toute anomalie thyroïdienne en début de grossesse; l'idéal étant bien évidemment une prise en charge de ces pathologies avant la conception [8].

Le but de notre étude est de déterminer la prévalence de la pathologie thyroïdienne au cours du premier trimestre de la grossesse et d'évaluer la fonction thyroïdienne durant ces premiers mois de gestation.

\section{Patients et méthodes}

\subsection{Etude}

Nous avons mené une étude prospective sur une période de 10 mois (de juillet 2016 à Mai 2017). Notre étude a concerné des gestantes au 1er trimestre de la grossesse consultant à la polyclinique de Oued Athmania (wilaya de Mila). Les femmes enceintes au deuxième et troisième trimestre ont été exclues de l'étude.

\section{2. Méthodes}

Le recueil des données nécessaires à l'étude a été effectué par l'interrogatoire des femmes enceintes selon un questionnaire préétabli. Un consentement éclairé à été établi pour toute la population d'étude.

\subsubsection{Prélèvements sanguins}

Les prélèvements sanguins ont été effectués par ponction veineuse au pli du coude, le matin à jeun chez toutes les gestantes. Le sang recueilli sur tubes contenant de l'héparinate de lithium a été adressé au laboratoire central de biochimie du Centre hospitalier universitaire (CHU) de Constantine.

Tous les tubes ont été rapidement centrifugés à $4000 \mathrm{tr} / \mathrm{mn}$ pendant $15 \mathrm{mn}$ et analysés le jour même.

\subsubsection{Dosages des paramètres biochimiques}

Toutes nos gestantes ont bénéficié d'un bilan thyroïdien qui a compté un dosage de la TSH, la fT4 et la fT3. Les trois paramètres ont été dosés sur un analyseur « Immulite ${ }^{2} 2000$ » des laboratoires Siemens ${ }$.

Méthode de dosage de la TSH

Le dosage de la TSH 3ème génération a été réalisé par une méthode quantitative par chimiluminescence immunométrique, en deux étapes en phase solide (Caractéristiques analytiques et valeurs de références (tableau I)).

Méthode de dosage de la fT3

Le dosage de la fT3 est un Immunodosage compétitif sur ana- logue (Caractéristiques analytiques et valeurs de références - tableau I)).

Méthode de dosage de la fT4

Le dosage de la fT4 a été effectué par une méthode d'immuno-analyse compétitive en phase solide utilisant la chimiluminescence avec marquage enzymatique en deux étapes (Caractéristiques analytiques et valeurs de références (tableau I)).

Tableau I. Caractéristiques analytiques et valeurs de références pour les méthodes de dosage utilisées.

\begin{tabular}{|c|c|c|c|c|c|}
\hline Paramètre & $\begin{array}{l}\text { Limite de } \\
\text { détection }\end{array}$ & $\begin{array}{l}\text { Sensibilité } \\
\text { analytiquee }\end{array}$ & $\begin{array}{l}\text { Domaine } \\
\text { de mesuree }\end{array}$ & Spécificitité & $\begin{array}{l}\text { Valeurs de } \\
\text { référence }\end{array}$ \\
\hline TSH(mU/L) & I & 0.004 & Jusqu'à 75 & Excellente & $0.4-4.0$ \\
\hline $\mathrm{fT4}$ (pmol/L) & 2.8 & 1.4 & $3.9-77.4$ & Excellente & $11.5-22.7$ \\
\hline $\mathrm{fT3}(\mathrm{pmol} / \mathrm{L})$ & I & 1.5 & $1.5-61.4$ & Excellente & $2.8-6.5$ \\
\hline
\end{tabular}

\subsection{Analyse statistique}

L'analyse statistique des données a été réalisée par le logiciel épi info version 7.0. La représentation graphique des résultats était réalisée sur Excel 2010.

\section{Résultats}

L'âge moyen de nos gestantes était de $30 \pm 6,76$ ans avec des extrêmes allant de 17 à 45 ans. L'âge gestationnel de nos femmes enceintes, variait entre 1 à 12 semaines d'aménorrhée avec une moyenne de 7,38 $\pm 3,37$ SA. 11 femmes étaient au 1 er mois (une à quatre semaines d'aménorrhée), 19 femmes au Zème (cinq à huit semaines d'aménorrhée) et 20 au 3ème mois de grossesse (neuf à treize semaines d'aménorrhée) (tableau II). Dans notre série, $80 \%$ de nos gestantes étaient en euthyroïdie clinique, $10 \%$ étaient suivies pour une hyperthyroïdie et $10 \%$ avaient une hypothyroïdie; diagnostiquées par des médecins spécialistes et traitées.

Les antécédents familiaux de pathologie thyroïdienne ont été retrouvés chez $10 \%$ de femmes enceintes, avec une hypothyroïdie dans $2 \%$ des cas, une hyperthyroïdie dans $4 \%$ des cas, un goitre dans $2 \%$ des cas et une thyroïdite d'Hashimoto dans $2 \%$ des cas également. Notre étude a retrouvé 11 pri- 
migestes soit 22\%; 10 femmes au 2ème geste soit 20\%; 10 femmes au 3ème geste soit 20\%; 9 femmes au 4ème geste soit $18 \% ; 7$ femmes soit $14 \%$ au 5 ème geste et 3 femmes soit $6 \%$ au 6ème geste. La plupart des femmes de notre étude se trouvent à leur 1er geste soit $22 \%$.

La TSH

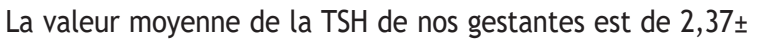
$4,01 \mathrm{mUI} / \mathrm{L}$ avec des extrêmes allant de 0,031 à $26,37 \mathrm{mUI} / \mathrm{L}$. Parmi les cinquante gestantes, on a noté une dysthyroïdie chez 15 d'entre elles soit 30\% des cas dont 12\% d'hypothyroïdie et $18 \%$ d'hyperthyroïdie. La répartition des gestantes selon la moyenne de la TSH et l'âge gestationnel a permis de constater que les concentrations de la TSH variaient d'une manière inversement proportionnelle à l'âge gestationnel. Le taux moyen de la TSH était élevé au premier mois de grossesse; intermédiaire au deuxième mois et relativement bas au troisième mois de la grossesse (Tableau II).

La fT3

Nos femmes enceintes présentaient une concentration plasmatique moyenne de $\mathrm{T} 3$ de $\quad 7,36 \pm 15,75 \mathrm{pmol} / \mathrm{L}$ avec des extrêmes allant de 1,02 à $100 \mathrm{pmol} / \mathrm{L}$. Parmi les cinquante gestantes, deux avaient un taux plasmatique de $\mathrm{T} 3<$ 2,8 $\mathrm{pmol} / \mathrm{L}$ soit $4 \%$ et vingt-et-un avaient un taux plasmatique de $\mathrm{T} 3>6,5 \mathrm{pmol} / \mathrm{L}$ soit $42 \%$. L'analyse des variations de la concentration moyenne de la fT3 en fonction de l'âge gestationnel a montré un taux élevé au premier mois de la grossesse, qui chutait au $1 / 3$ de sa valeur aux deuxième et troisième mois de grossesse (tableau II).

La fT4

La concentration plasmatique moyenne de la fT4 dans notre série était de 17,204 $\pm 9,023 \mathrm{pmol} / \mathrm{l}$, avec des extrêmes allant de 7,53 à 66,43pmol/l. Parmi les cinquante gestantes, six avaient un taux plasmatique de fT4<11,5 pmol// soit 12 $\%$ et cinq avaient un taux plasmatique de fT4 $>22,7 \mathrm{pmol} / /$ soit $10 \%$. La comparaison des concentrations plasmatiques moyennes de la fT4 en fonction de l'âge gestationnel a mis en évidence une augmentation de la moyenne du taux plasmatique de la fT4 au premier mois, puis une légère diminution de celle-ci au deuxième et troisième mois, tout en restant dans l'intervalle des valeurs normales (tableau II).

L'analyse des variations de la concentration plasmatique des hormones thyroïdiennes au cours du premier trimestre de la grossesse, a montré une diminution de la concentration plasmatique de la TSH en passant du 1er mois au 3ème mois de la grossesse. Les deux autres paramètres du bilan thyroïdien ( $\mathrm{fT} 3, \mathrm{fT} 4$ ) présentaient une élévation de leurs concentrations au premier mois puis une stabilisation au 2ème et 3ème mois de grossesse (figure 1).
Figure 1: Variation de la concentration plasmatique des hormones thyroïdiennes au cours du premier trimestre de la grossesse

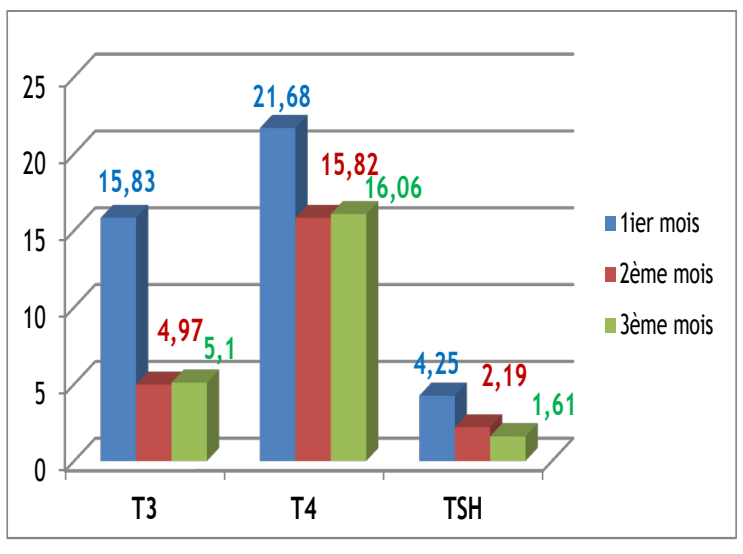

Parmi les $20 \%$ de gestantes présentant une dysthyroïdie connue et traitée, $8 \%$ étaient en euthyroïdie (bien équilibrées) et $12 \%$ étaient toujours en dysthyroïdie malgré le traitement (non équilibrées).

-Résumé des caractéristiques des gestantes de notre série

Tableau II: Résumé des caractéristiques des gestantes de notre série

\begin{tabular}{|c|c|}
\hline Paramètres & Total $(n=50)$ \\
\hline Age (ans) & 17 à 45 ans. \\
\hline Age gestationnel (mois) & \\
\hline $1^{\mathrm{er}}$ mois, & 11 femmes enceintes \\
\hline $2^{\text {ème }}$ mois & 19 femmes enceintes \\
\hline $3^{\text {ème }}$ mois & 20 femmes enceintes \\
\hline \multicolumn{2}{|l|}{$\begin{array}{l}\text { ATCD personnels thyroïdiens } \\
\text { de notre série }\end{array}$} \\
\hline Euthyroïdie clinique & $80 \%$ \\
\hline Hyperthyroïdie & $10 \%$ \\
\hline Hypothyroïdie & $10 \%$ \\
\hline \multicolumn{2}{|l|}{ ATCD familiaux thyroïdiens } \\
\hline de notre série & $10 \%$ des femmes enceintes \\
\hline Hypothyroïdie & $2 \%$ des cas \\
\hline Hyperthyroïdie & $4 \%$ des cas \\
\hline Goitre & $2 \%$ des cas \\
\hline Thyroïdite d'Hashimoto & $2 \%$ des cas \\
\hline \multicolumn{2}{|l|}{ Résultats biologiques } \\
\hline TSH & \\
\hline $1^{\mathrm{er}}$ mois & $4,2470 \pm 8,2199 \mathrm{mUl} / \mathrm{L}$ \\
\hline 2ème mois & $2,1889 \pm 2,1093 \mathrm{mUI} / \mathrm{L}$ \\
\hline 3ème mois & $1,6008 \pm 1,3583 \mathrm{mUl} / \mathrm{L}$ \\
\hline \\
\hline $1^{\mathrm{er}}$ mois & $15,820 \pm 34,040 \mathrm{pmol} / \mathrm{L}$ \\
\hline $2^{\text {ème }}$ mois & $4,973 \pm 1,933 \mathrm{pmol} / \mathrm{L}$ \\
\hline $3^{\text {ème }}$ mois & $5,095 \pm 1,454 \mathrm{pmol} / \mathrm{L}$ \\
\hline \multicolumn{2}{|l|}{ T4 } \\
\hline $1^{\mathrm{er}}$ mois & $21,6791 \pm 17,7811 \mathrm{pmol} / \mathrm{L}$ \\
\hline $2^{\text {ème }}$ mois & $15,8168 \pm 4,2202 \mathrm{pmol} / \mathrm{L}$ \\
\hline $3^{\text {ème }}$ mois & $16,0610 \pm 3,4357 \mathrm{pmol} / \mathrm{L}$ \\
\hline
\end{tabular}




\section{Discussion}

Dans ce travail, nous avons étudié les variations des concentrations plasmatiques de la fT3, fT4 et la TSH chez cinquante gestantes au 1ertrimestre de la grossesse. Toutes nos gestantes sont originaires de l'Est algérien. La prévalence de l'hypothyroïdie dans notre série est $8 \%$ ce qui est plus élevé que la prévalence des cas rapportés chez les femmes enceintes européennes $2,2 \%$, américaines $2,5 \%[5,9,10,11$, 12,13 ] japonaise $0,3 \%$ [4] et tunisiennes 3,2\% [14]. Nos résultats sont cependant proches de ceux de l'étude marocaine qui a retrouvé $6,52 \%$ cas d'hypothyroïdie [2]. La carence en iode est la première cause d'hypothyroïdie dans le monde [16, 17]. Dans les zones où l'apport en iode est suffisant, telles qu'aux Etats-Unis ou en Europe, l'étiologie la plus fréquente de l'hypothyroïdie est la thyroïdite chronique auto-immune (Thyroïdite d'Hashimoto) [17]. Dans notre étude, la thyroïdite d'Hashimoto n'a pas été retrouvée, il s'agit probablement d'une carence iodée.

Tableau III: Fréquence des hypothyroïdies chez la femme enceinte selon les auteurs.

\begin{tabular}{|l|c|c|}
\hline \multicolumn{1}{|c|}{ Pays } & fréquence & Série \\
\hline Série européenne $^{[10]}$ & $2,2 \%$ & - \\
\hline Série américaine $^{[9,18]}$ & $2,5 \%$ & - \\
\hline Série tunisienne $^{[14]}$ & $3,2 \%$ & 1519 \\
\hline Série marocaine $^{[2]}$ & $6,52 \%$ & 432 \\
\hline Notre Série & $\mathbf{8} \%$ & $\mathbf{5 0}$ \\
\hline
\end{tabular}

Selon la littérature, l'hyperthyroïdie serait présente chez 1 à $3 \%$ des femmes enceintes [19, 20].

Dans notre étude, l'hyperthyroïdie a été observée chez $10 \%$ de gestantes. Nos résultats sont élevés par rapport aux données de la littérature et l'étude marocaine qui a retrouvé $3,47 \%$ cas d'hyperthyroïdie. Cette discordance dans les résultats peut être due à nos critères de sélection (premier trimestre de la grossesse) d'une part, et à la petite taille de notre échantillon d'autre part.

Concernant la relation de la concentration plasmatique de la TSH avec l'âge gestationnel, nous avons retrouvé une évolution inversement proportionnelle. La concentration plasmatique de la TSH diminue à mesure que l'âge gestationnel augmente. Elle est plus élevée au premier mois $(4,25 \pm 8,22 \mathrm{mUl} / \mathrm{L})$, intermédiaire au deuxième mois $(2,19 \pm 2,109 \mathrm{mUl} / \mathrm{L})$ et basse au troisième mois $(1,60 \pm 1,36 \mathrm{mUI} / \mathrm{L})$. Nos résultats sont similaires à ceux de Guillaume J et al [21], Pekonen F et al [22] et Ballabio $M$ et al [23], qui ont montré qu'à partir de la 6ème semaine de gestation, la concentration plasmatique de TSH suit une courbe inverse de celle de l'hCG qui culmine à la 12èmesemaine [5]. Cette diminution de la TSH au début de la grossesse est imputable à l'augmentation de l'hCG au 1er trimestre [24, 25]. L'hCG synthétisée par le placenta exerce un effet thyréostimulant sur la thyroïde maternelle du fait de son homologie structurale avec la TSH (sous-unité alpha). Elle se lie au récepteur de la TSH et stimule par conséquent la croissance de l'épithélium thyroïdien et les différentes étapes de la production hormonale. L'élévation du taux des hormones thyroïdiennes exerce un rétrocontrôle négatif sur l'hypophyse ce qui explique la chute de la TSH [26-29]. Les deux autres paramètres du bilan thyroïdien (fT3, fT4) présentent une élévation de leurs concentrations au premier mois puis une stabilisation au 2ème et 3ème mois de la grossesse.

Nos résultats rejoignent ceux de plusieurs études qui montrent que la grossesse entraine une augmentation de la fonction thyroïdienne. Cette augmentation de l'activité fonctionnelle de la glande thyroïde maternelle est due à l'action TSH-like de l'hormone chorionique gonadotrophique (hCG), l'apparition de l'activité de la désiodase placentaire de type 3 et l'élévation de la concentration plasmatique de la TBG (thyroxine-binding globulin). Cette dernière est secondaire à la stimulation de sa synthèse hépatique par l'hyperœstrogénie [30,31]. La capacité de fixation pour la T3 et la T4 est ainsi accrue. Les concentrations des formes totales d'hormones thyroïdiennes (T4, T3) augmentent, très précocement dès le début de la grossesse, et resteront élevées durant les six mois qui suivent l'accouchement. En revanche, il y a peu de modification des concentrations des formes libres (fT4, fT3) qui restent sensiblement normales tout au long de la grossesse, avec quand même une réduction relative des concentrations hormonales (de l'ordre de 20 à $30 \%$ ) du fait de l'hémodilution et de la carence en iode [26].

Il convient de signaler par ailleurs, le passage transplacentaire de l'hormone T4 en début de grossesse, en particulier durant la période où la thyroïde fœtale n'est pas fonctionnelle (la concentration d'iode par la thyroïde fœtale commence à 12 SA et le fonctionnement sous la dépendance de la TSH est acquis à $20 \mathrm{SA})$ [32]. 


\section{Conclusion}

Même si elle ne s'exprime pas avec éloquence, la thyroïde est soumise à une rude épreuve au cours de la grossesse. Les dysthyroïdies, fréquentes chez la femme jeune, peuvent évoluer de façon variable en cours de grossesse et exposent, lorsqu'elles sont négligées ou mal appréciées, à des complications materno-fœtales pouvant être sévères. Leur prise en charge doit être précoce, adéquate, et nécessite une collaboration étroite entre endocrinologue et obstétricien.

Au terme de cette étude, II serait plus judicieux de faire d'autres études sur un nombre plus important de gestantes et d'inclure d'autres paramètres biologiques pour une meilleure interprétation des résultats.

Un certain nombre de recommandations pratiques doit cependant être formulé:

- Il faut favoriser l'apport en iode aux femmes enceintes. Il est toutefois préférable de corriger une éventuelle carence iodée avant le début de la grossesse.

- La surveillance de la TSH est hautement souhaitable avant la conception, ou au moins au 1er trimestre de la grossesse en cas de goitre, d'antécédent personnel ou familial de pathologie thyroïdienne.

- L'augmentation de la posologie du traitement hormonal d'environ $30 \%$ est à recommander dès le début de la conception, chez les hypothyroïdiennes soumises au traitement substitutif

\section{Conflits d'intérêt}

Les auteurs déclarent n'avoir aucun conflit d'intérêt.

\section{Références bibliographiques}

[1]. ACOG Practice Bulletin. Clinical management guidelines for obstetrician-gynecologists. No 37, août 2002. Thyroid disease in pregnancy. Obstet Gynecol 2002; 100(2): 387-96.

[2].Lahlou Kenza. Dysthyroïdie et grossesse. Mémoire pour l'obtention du diplome de spécialité en médecine option: endocrinologie et maladies métaboliques. session juin 2015.

[3]. Hind Iraki.Thyroïde et grossesse. Magazine professionnel d'information médicale. Doctinews N 55 Mai 2013.

[4] .V. Rohmer. Goitres et troubles de la fonction thyroïdienne chez la femme enceinte. Annales d'Endocrinologie, Vol 63, № 5 - octobre 2002 pp. 432-437.

[5]. Glinoer, D. The regulation of thyroid function in pregnancy: pathways of endocrine adaptation from physiology to pathology. Endocr Rev, 1997. 18(3): p. 404- 33.

[6]. Taura, M, M. Izumi, and S. Nagataki. Release of thyroid hormone from circulating thyroglobulin in the rat. Acta Endocrinol (Copenh), 1986. 111(2): p. 209-12.3.

[7]. Haddow JU, Palomaki GE, Allan WC, Williams JR, Knight GJ, Gagnon J, O'Heir CE, Mitchell ML, Hermos RJ, Waisbren SE, Faix JD, Klein RZ. Maternal thyroid deficiency during pregnancy and subsequent neuropsychological development of the child. N. Engl J Med 1999, 341, 549-555.

[8]. Shupnik, M. A, E. C. Ridgway, and W.W. Chin, Molecular biology of thyrotropin.Endocr Rev, 1989. 10(4): p. 459-75.

[9].Casey BM, Dashe JS, Wells CE, McIntire DD, Byrd W, Leveno KJ et al. Subclinical hypothyroidism and pregnancy outcomes. Obstet Gynecol 2005; 105:239-45.

[10]. Millar LK, Wing DA, Leung AS, Koonings PP, Montoro MN, Mestman $\mathrm{JH}$. Low birth weight and preeclampsia in pregnancies complicated by hyperthyroidism. Obstet Gynaecol 1994; 84: 946-9.

[11].Bray GA. Increased sensitivity of the thyroid in iodinedepleted rats to the goitrogenic effects of thyrotropin. J Clin Invest 1968; 47:1640-7.

[12].Vulsha T, Gons MH, de Vijlder JJM. Maternal-fetal transfer of thyroxine in congenital hypothyroidism due to a total organification defect or thyroid agenesis. N Engl J Med 1989; 321: 1316.

[13].Stagnaro-Green, A, et al. Guidelines of the American Thyroid Association for the diagnosis and management of thyroid disease during pregnancy and postpartum. Thyroid, 2011. 21(10): p. 1081-125.

[14].Leung AS, Millar LK, Koonings PP, Montoro M, Mestman JH. Perinatal outcomein hypothyroid pregnancies. ObstetGynaecol 1993; 81: 349-53.

[15].Glinoer, D. What happens to the normal thyroid during pregnancy? Thyroid, 1999. 9(7): p. 631-5.

[16].Thorpe-Beeston JG, Nicolaides KH, Felton CV, Butler J. McGregor AM. Maturation of the secretion of thyroid hormone and thyroid-stimlating hormone in the fetus. N Engl J Med 1991; 324: 532-536.

[17]. Stagnaro-Green A. Approach to the patient with postpartum thyroiditis. The journal of clinical endocrinology and metabolism 2012; 97(2): 334-342.

[18].Glinoer D, Riahi M, Grun JP, Kinthaert J. Risk of subclinical hypothyroidism in pregnant women with asymptomatic autoimmune thyroid disorders. J Clin Endocrinol Metab 1994; 79: 197-204.

[19].Gaberscek S, Zaletel K. Thyroid physiology and autoimmunity in pregnancy and after delivery. Expert review of clinical immunology 2011; 7(5): 697-707.

[20].Mestman JH. Hyperthyroidism in pregnancy.Endocrinol Metab Clin North Am1998, 27: 127-149. 
[21]. Guillaume J, Schussler GC, Goldman J. Components of the total serum thyroid hormone concentrations during pregnancy: high free thyroxine and blunted thyrotropin (TSH) response to TSH-releasing hormone in the first trimester. J Clin Endocrinol Metab1985; 60: 678-684 121.

[22]. Pekonen F, Alfthan H, Stenman U-H, Ylikorkala O. Human chorionic gonadotropin (hCG) and thyroid function in early human pregnancy: circadian variation and evidence for intrinsic thyrotropic activity of hCG. J ClinEndocrinolMetab 1988; 66: 853-856 122.

[23]. Ballabio M, Poshyachinda M, Ekins RP. Pregnancy-induced changes in thyroid function: role of human chorionic gonadotropin as putative regulator of maternal thyroid. J Clin Endocrinol Metab 1991; 73: 824-831.

[24]. Defossez A, Maurage C.A, Caratero. Histologie humaine, chap14: Systéme endocrine juillet 2006; $3:$ 283-293.

[25]. Ph. Mauclet. Lathyroïde, la grossesse et le fœtus.Belgian Thyroid Club 34th meeting, Diegem, mai 2009.

[26].Jean-Luc Wemeau. Thyroïde et grossesse. Journées Pyrénéennes de Gynécologie - Tarbes - 3 \& 4 octobre 2003.

[27].Glinoer D, DeNayer P,Bourdoux P et al. Regulation of maternal thyroid during pregnancy. J Clin Endocrinol Metab.1990, 71: 276-287.

[28].Godeau P, BletryO, Garin JL et al. Hyperthyroidie par choriocarcinome placentaire. Ann Med Interne.1980, 131: 233-227.

[29]. Sophie Alexander, Patricia Barlow, Pierre Bernard, Gilles Ceysens, Maïté Delfosse, et al. Thyroïde et grossesse. Groupement des Gynécologues Obstetriciens de Langue Francaise de Belgique. 2017.

[30]. Françoise Borson-Chazot, Philippe Caron. Thyroïde et grossesse. Médecine Clinique endocrinologie \& diabète n 86 - Janvier 2017.

[31]. C. Bournaud, J. Orgiazzi. Thyroïde et grossesse. Annales d'EndocrinologieVol 64, № 4 - octobre 2003pp. 324-331.

[32]. Sophi-Gariepy Major D.O. Troubles Thyroïdiens. Diagnostique et surveillance biologique des maladies thyroidiennes. 2007 1-3. 
